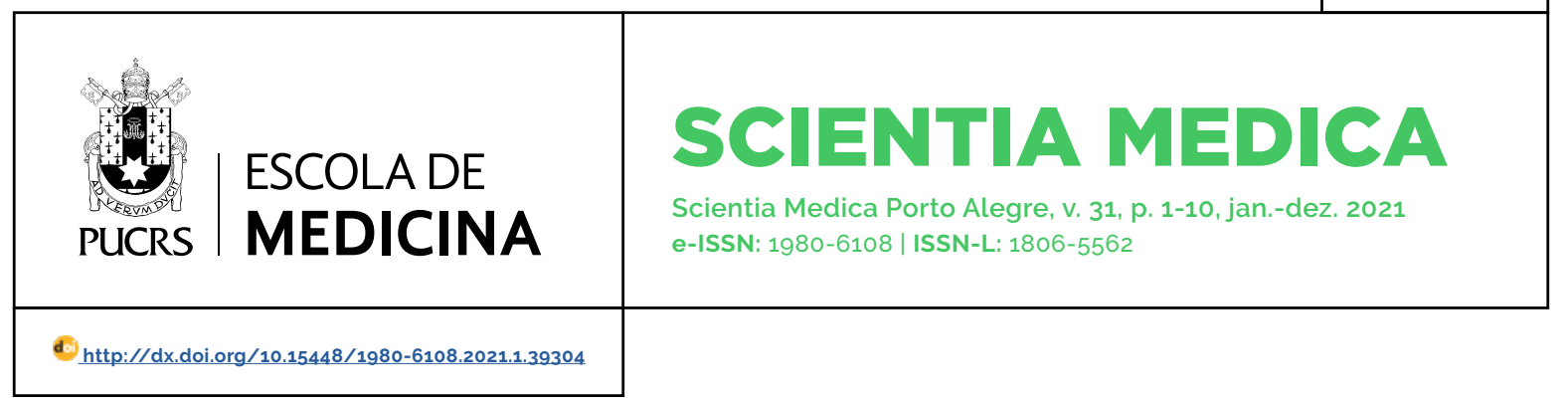

ARTIGO ORIGINAL

\title{
A utilização da acupuntura no controlo da dor crónica em usuários com síndrome vertebral com irradiação
}

\author{
The use of acupuncture to control chronic pain in users with irradiated vertebral syndrome
}

\author{
Elisabete Fernandes \\ Carvalho ${ }^{1}$ \\ orcid.org/0000-0001-9585-0507 \\ elicar258@gmail.com
}

Dinis Rodrigues Brito ${ }^{2}$

orcid.org/0000-0002-7547-0053

dr.dinisbrito@gmail.com

\section{Isabel Maria Antunes \\ Rodrigues da Costa \\ Barroso $^{3}$ \\ orcid.org/0000-0001-5112-6015 \\ imbarroso@utad.pt}

\section{Maria João Filomena dos Santos Pinto \\ Monteiro 4 \\ orcid.org/0000-0003-0610-0670 \\ mjmonteiro@utad.pt}

\section{Vitor Manuel Costa \\ Pereira Rodrigues 5}

orcid.org/0000-0002-2795-685X

vmcpr@utad.pt

Recebido em: 5 out. 2020. Aprovado em: 26 abr. 2021 Publicado em: 17 jun. 2021.

\section{(c) (i)}

Artigo está licenciado sob forma de uma licença Creative Commons Atribuição 4.0 Internacional.

\section{Resumo}

Objetivos: a acupuntura é, cada vez mais, uma técnica utilizada, em usuários portadores de várias patologias, e, em especial, aquelas que são acompanhadas de dor crónica. Esta pesquisa teve como objetivo geral analisar o efeito da terapêutica não farmacológica de acupuntura e eletroacupuntura para o controle da dor crónica, em usuários com diagnóstico médico de sindrome vertebral com irradiação. Métodos: foi realizada uma pesquisa com dois momentos de avaliação - antes e após sessões de terapêutica não farmacológica de acupuntura e eletroacupuntura. Utilizou-se um questionário com variáveis de caracterização sociodemográfica e clínica, o Brief Pain Inventory (BPI) e Short Form 6 Dimensions (SF-6D).

Resultados: participaram no estudo 43 usuários, tendo sido encontradas diferenças estaticamente significativas, do momento 1 para o momento 2 de avaliação, quer no domínio da severidade da dor $(p<0,01)$, quer no domínio interferência da dor no funcionamento diário $(p<0,01)$, assim como nas diferentes dimensões do SF-6D foram encontradas diferenças estatisticamente significativas.

Conclusões: salienta-se que a terapêutica não farmacológica de acupuntura e eletroacupuntura em usuários com diagnóstico de sindrome vertebral com irradiação e dor crónica contribuiu para a diminuição da severidade da dor, da interferência da dor no funcionamento diário e melhoria da qualidade de vida relacionada com a saúde.

Palavras-chave: terapia por acupuntura, dor crônica, qualidade de vida.

\section{Abstract}

Aims: acupuncture is increasingly a technique used in users with various pathologies, and especially those who are accompanied by chronic pain. This research aimed to analyze the effect of non-pharmacological acupuncture and electroacupuncture therapy for the control of chronic pain in users with medical diagnosis of irradiation vertebral syndrome.

Methods: a research was conducted with two evaluation moments: before and after non-pharmacological acupuncture and electroacupuncture therapy sessions. A questionnaire with variables of sociodemographic and clinical characterization, the Brief Pain Inventory and Short Form 6 Dimensions (SF-6D) was used.

Results: forty-three users participated in the study, and statistically significant differences were found from moment 1 to moment 2 of evaluation, either in the pain severity domain $(p<0.01)$ or in the pain interference domain in daily functioning $(p<0.01)$, as well as in the different dimensions of SF-6D, statistically significant differences were found.

Conclusions: non-pharmacological acupuncture and electroacupuncture therapy in users diagnosed with irradiation and chronic pain have contributed to decrease pain severity, pain interference in daily functioning and improvement of health-related quality of life.

\footnotetext{
Unidade de Saúde Pública: Agrupamento de Centros de Saúde do Cávado III - Barcelos/Esposende, Barcelos, Portugal.

Unidade de Saúde Familiar 7 Fontes, Agrupamento de Centros de Saúde Cávado I - Braga, Braga, Portugal.

Universidade de Trás-os-Montes e Alto Douro (UTAD), Escola Superior de Saúde, Vila Real, Portugal.

Centro de Investigação em Tecnologias e Serviços de Saúde (CITSS), Universidade de Trás-os-Montes e Alto Douro (UTAD), Escola Superior de Saúde, Vila Real, Portugal.

5 Centro de Investigação em Desporto, Saúde e Desenvolvimento Humano (CIDESD), Universidade de Trás-os-Montes e Alto Douro (UTAD), Escola Superior de Saúde, Vila Real, Portugal.
} 
Keywords: acupuncture therapy, chronic pain, quality of life.

ABREVIATURAS: BPI, Inventário Resumido da Dor (Brief Pain Inventory); SF-6D, questionário Short Form 6 Dimensions.

\section{Introdução}

A dor é um fenómeno fisiológico que compromete a integridade física e constitui o principal motivo para a procura de cuidados de saúde por parte da população em geral. Percebemos a dor, como objeto de análise, a partir da definição da International Association for the Study of Pain que definiu a dor como uma experiência sensorial ou emocional desagradável associada à lesão tecidual, real ou potencial, ou descrita em termos de tal lesão (1).

A dor induz sofrimento, reduz a qualidade de vida, condiciona a gestão da vivência quotidiana, provoca alterações fisiopatológicas que vão contribuir para o aparecimento de comorbilidades orgânicas e psicológicas e perpetuar o fenómeno doloroso. É, por isso, considerada um fenómeno corpo-mente. De facto, uma dor intensa pode provocar sofrimento, e o sofrimento, pela sua intensidade, pode provocar dor, causando um impacto na pessoa muito para além do sofrimento que the causa, nomeadamente, sequelas psicológicas, isolamento, incapacidade e perda de qualidade de vida, que pode ultrapassar a própria pessoa e envolver a família, cuidadores e amigos. Tendo em conta que se trata de uma experiência subjetiva, não há testes objetivos para medir a presença ou a intensidade da dor, devendo ser avaliadas pelo que o doente exprime, tornando-se essencial investir em estudos nos quais se possa não só investigar a intensidade da dor, como também a sua implicação na vida das pessoas, uma vez que as informações verbalizadas sobre a dor devem ser sempre valorizadas, sendo o paciente o único que pode referir sobre a intensidade, o padrão e a natureza da dor e que consegue descrevê-la da forma como é sentida (2).

Atualmente, a avaliação da dor nos doentes é um critério obrigatório, por se tratar de um sintoma prevalente na duração da maioria das doenças, sendo que essa avaliação compreende o exame clínico, as caracteristicas da experiência de dor, as repercussões da dor nas atividades de vida diárias e a investigação de elementos psiquicos e socioculturais significativos.

A dor, mais concretamente a dor crónica, tem impacto no ser humano, transcendendo o sofrimento que the causa, sobretudo, sequelas psicológicas, isolamento, incapacidade e perda de qualidade de vida, podendo ultrapassar a própria pessoa e envolver a familia, cuidadores e amigos, sendo também significantes as repercussões socioeconómicas pelos custos envolvidos no recurso frequente aos serviços de saúde e despesas com a terapêutica, sendo certo que a dor crónica, ao persistir para além da cura da lesão, deve ser encarada não como um sintoma, mas sim como uma doença só por si (3).

Neste contexto, a lombalgia é definida como uma dor e desconforto, tipicamente localizada na região posterior, entre a margem inferior das últimas costelas e as pregas glúteas, sendo frequentemente acompanhada por dor em um ou ambos os membros inferiores, sendo a dor lombar crónica uma condição médica complexa, heterogénea que abrange uma vasta multiplicidade de sintomas, sendo uma causa frequente de morbidade e de incapacidade (4). Contudo, uma historia clinica que refira a existência de dor que irradia para o membro inferior, o diagnóstico clínico passa a ser lombociatalgia, que pode ser de origem radicular (exemplo: compressão por hérnia de disco) ou referida (exemplo: dor miofascial), tendo como principais etiologias a protrusão discal, a hérnia de disco, a estenose de canal vertebral, a sindrome pós-laminectomia e a sindrome do piriforme (5). Nos pacientes com perturbações do sistema músculo-esquelético, especialmente nos membros inferiores e na região lombar, uma das consequências mais frequentes é a imobilidade física, motivando grave comprometimento na realização das atividades de vida diárias, resultando num elevado grau de dependência, conduzindo a um grande desafio (6), até porque, em âmbito mundial, a dor crónica traduz uma tendência cada vez mais crescente.

Os métodos de tratamento não farmacológicos 
de controle da dor poderão ser uma alternativa terapêutica equilibrada, segura, bem tolerada e custo-efetiva e, em associação a fármacos, potencializar o efeito terapêutico global, reduzindo as doses farmacológicas e minimizando os efeitos colaterais e adversos (7).

No que concerne às terapêuticas não convencionais para o alivio da dor, o seu avanço tem vindo a afirmar-se como um tratamento não farmacológico coadjuvante, entre as quais se destaca a acupuntura. A acupuntura tem sido uma das terapêuticas utilizadas em inúmeras e variadas situações de dor (8). Trata-se de uma prática tradicional chinesa cujo objetivo é o de promover a cura pela estimulação do equilibrio do corpo e atuação das energias negativas e positivas (9). Consiste na inserção manual de agulhas para estimular terminações nervosas mecanossensiveis. Já a eletroacupuntura, recorre a estímulos elétricos aplicados nas agulhas, atuando em todos os tipos de fibras. Essa técnica é classificada como apropriada no tratamento da dor crónica devido às suas reações neurobiológicas, que intervêm a nivel dos neurotransmissores relacionados com a dor e com a depressão (9).

Na terapêutica da dor crónica, as principais indicações da acupuntura centram-se na dor nociceptiva do tipo músculo-esquelético com carateristica inflamatória ou não, com principais benefícios na lombalgia, cervicalgia, omalgia, osteoartrite do joelho e cefaleia crónica (10).

A Organização Mundial da Saúde recomenda e estimula o uso desta terapia, a fim de promover uma prática segura e incentivar a sua integração nos sistemas de saúde dos vários países em âmbito mundial e o exercício mútuo de ambas as medicinas, em complementaridade, em prol da maximização dos benefícios em saúde para os usuários (11).

Tendo por base que a abordagem das pessoas com dor é possivel e deve ser baseada na melhor evidência científica, no sentido de prevenir e controlar a dor, melhorar a sua qualidade de vida e capacidade funcional (3), foi elaborada esta pesquisa que teve como objetivo geral analisar o efeito da terapêutica não farmacológica de acu- puntura e de eletroacupuntura para o controle da dor crónica, em usuários com diagnóstico médico de sindrome vertebral com irradiação e como objetivos específicos verificar se a terapêutica não farmacológica de acupuntura e de eletroacupuntura contribui para a diminuição da severidade da dor, para a diminuição da interferência da dor no funcionamento diário do indivíduo, para a melhoria da qualidade de vida e para a diminuição dos custos diretos em saúde (medicação e tratamentos relacionados com a dor).

\section{Métodos}

Tratou-se de uma pesquisa descritiva, correlacional e longitudinal, com dois momentos de avaliação: antes e após sessões de terapêutica não farmacológica de acupuntura e de eletroacupuntura, realizada entre 15 de dezembro de 2016 a 3 de abril de 2017. Os usuários foram posicionados comodamente em decúbito ventral e submetidos, de modo análogo, à puntura com agulhas de acupuntura estéreis de uso único $(0,25 \times 25 \mathrm{~mm}$ e $0,32 \times 100 \mathrm{~mm})$, inseridas perpendicularmente em locais anatómicos especíicos e precisos, que foram estimuladas manualmente durante pelo menos 10 segundos. Na acupuntura manual, os pontos de acupuntura, previamente selecionados segundo os princípios padronizadas e descritos pela Medicina Tradicional Chinesa $(12,13)$, incluiram: Shenshu (B-23), Da Changshu (B-25), Chengfu (B36), Yinmen (B-37), Weizhong (B-40), Kunlun (B-60), Shenmai (B-62), Yaoyanguan (Du-3), Mingmen (Du-4) e o Tunzhong (ponto extra), e, em seguida, emparelhou-se os elétrodos do equipamento de eletroacupuntura (estimulador elétrico de pontos acupuntura) às asas das agulhas nos pontos B-23 e B-25, com baixa frequência de 2 a $5 / 100 \mathrm{~Hz}$ e com a intensidade estabelecida pelo usuário perante o limiar de dor, durante cerca de 20 minutos.

A cada usuário, num primeiro momento, imediatamente antes da intervenção, foi aplicado o questionário de caracterização sociodemográfica e clínica, o Brief Pain Inventory (BPI) (14) e Short Form 6 Dimensions (SF-6D) (15). Imediatamente após, em cada usuário, fazia-se a execução da primeira intervenção, perfazendo o total de três 
semanas (uma vez por semana). Num segundo momento de avaliação, imediatamente após a terceira intervenção, a cada usuário foi novamente aplicado o BPI, o SF-6D e o questionário clínico.

A população que esteve na base deste estudo foi constituída por 43 usuários com diagnóstico médico de síndrome vertebral com irradiação com dor crónica pertencentes a uma unidade de saúde familiar da região Norte de Portugal. Os usuários foram contatados via telefone, durante a consulta médica ou de enfermagem, e convidados a participar na investigação de acordo com os seguintes critérios de inclusão: i) diagnóstico médico de síndrome vertebral com irradiação com dor crónica; ii) idade maior ou igual a 18 anos; iii) sem défices neurológicos; iv) participação voluntária no estudo e assinatura por escrito do consentimento informado. Foram considerados os seguintes critérios de exclusão: ter pacemaker e no caso de gravidez.

Como instrumentos de coleta de dados foram utilizados: i) um questionário com questões de natureza sociodemográfica e clínica; ii) o inventário resumido para a dor, que consiste num questionário de autopreenchimento e de autoavaliação das características da dor, constituído por 15 itens, sendo: i) um item dicotómico para verificação da existência da dor; ii) um item para localização da dor, através da indicação das áreas da dor num diagrama representativo do corpo humano; iii) uma escala de intensidade da dor constituida por quatro itens (máximo, mínimo, em média e no momento da avaliação) numa classificação de zero a 10 (zero não ter dor e 10 corresponde à pior dor que se pode imaginar); iv) um item para registo das estratégias terapêuticas aplicadas; v) um item que quantifica em termos percentuais a eficácia dos tratamentos aplicados; e vi) uma escala de interferência funcional constituida por sete itens numa classificação que varia de zero a 10 (zero corresponde a dor que não interfere com o funcionamento diário e 10 corresponde ao facto de a dor interferir completamente com o funcionamento diário), que avalia a interferência da dor nas atividades gerais, humor, mobilidade, trabalho, relações pessoais, sono e prazer de viver; e vii) o Questionário de avaliação de qualidade de vida relacionada com a saúde, que é um instrumento que mede a perceção do estado de saúde e da qualidade de vida através de seis dimensões: 1) função física; 2) limitação no desempenho; 3) função social; 4) dor física; 5) saúde mental; e 6) vitalidade. Esses domínios ou dimensões têm entre 4 a 6 niveis, em que o valor 1 representa o valor mínimo e os valores 4 , 5 ou 6 , dependendo da variável, representam os valores máximos. As dimensões função física e dor física apresentam 6 niveis cada e a função social, a saúde mental e a vitalidade apresentam 5 niveis e a limitação no desempenho apresenta 4 níveis. A escala apresenta um valor contínuo entre 0,30 a 1,00, na qual 1,00 significa "saúde plena".

A pesquisa foi autorizada pela Comissão de Ética para a Saúde da Administração Regional de Saúde do Norte (parecer n. ${ }^{\circ}$ 143/2016) e as pessoas envolvidas foram informadas e esclarecidas acerca da natureza e dos objetivos do estudo e convidadas a participar de forma voluntária, assinando um termo de consentimento informado, sendo assegurado o total anonimato e confidencialidade das informações obtidas.

Para edição e tratamento estatístico foi utilizado o programa de análise estatística IBM SPSS Statistics 22. Foi utilizada a estatística descritiva, nomeadamente frequências absolutas e relativas, médias e desvio padrão, e a estatística inferencial, nomeadamente o teste $t$ de Student, uma vez que se verificou os pressupostos de normalidade das variáveis, com um nivel de significância de 5\%.

\section{Resultados}

A amostra foi constituida 43 usuários com diagnóstico de sindrome vertebral com irradiação e dor crónica, com média de idade de 51,9 $\pm 10,5$ anos, sendo a idade mínima de 26 anos e a idade máxima de 77 anos, sendo o grupo etário entre os 45-64 anos o que apresentava maior percentagem (65,1\%). Conforme se pode verificar na Tabela 1, $29(67,4 \%)$ dos usuários são do sexo feminino, a maioria dos usuários $(65,1 \%)$ eram casados(as)/união de facto e a maioria (53.5\%) trabalhava a tempo inteiro. 
TABELA 1 - Caracterização sociodemográfica dos usuários de uma unidade de saúde familiar da região Norte de Portugal, 2017.

\begin{tabular}{|c|c|c|}
\hline Variáveis & $\mathrm{n}$ & $\%$ \\
\hline \multicolumn{3}{|l|}{ Género } \\
\hline Feminino & 29 & 67,4 \\
\hline Masculino & 14 & 32,6 \\
\hline \multicolumn{3}{|l|}{ Estado civil } \\
\hline Solteiro & 3 & 7,0 \\
\hline Casado/União de fato & 28 & 65,1 \\
\hline Separado/Divorciado & 9 & 20,9 \\
\hline Viúvo & 3 & 7,0 \\
\hline \multicolumn{3}{|l|}{ Escolaridade } \\
\hline $1^{\circ}$ Ciclo incompleto & 1 & 2,3 \\
\hline $1^{\circ}$ Ciclo & 10 & 23.3 \\
\hline $2^{\circ}$ Ciclo & 8 & 18,6 \\
\hline $3^{\circ}$ Ciclo & 3 & 7,0 \\
\hline Secundário & 12 & 27.9 \\
\hline Ensino Superior & 9 & 20,9 \\
\hline \multicolumn{3}{|l|}{ Atividade laboral } \\
\hline Trabalha a tempo inteiro & 23 & 53.5 \\
\hline Trabalha a tempo parcial & 1 & 2,3 \\
\hline Desempregado & 4 & 9.3 \\
\hline Doméstica & 2 & 4.7 \\
\hline Reformado/Pensionista & 6 & 14,0 \\
\hline Outro (baixa médica) & 7 & 16,3 \\
\hline
\end{tabular}

No que respeita à avaliação da qualidade de vida relacionada com a saúde apresentam-se, na Tabela 2, os resultados relativamente ao primeiro momento e ao segundo momento, isto é, antes e após a intervenção com a terapêutica não farmacológica de acupuntura e eletroacupuntura. Os resultados mostram que, em relação aos 43 usuários, as médias das seis dimensões do SF-6D apresentam valores mais elevados no primeiro momento de avaliação relativamente ao segundo momento. Assim, no primeiro momento, e de modo mais particular, a dimensão dor física é a que apresenta média mais elevada (4,72), correspondendo a pior saúde, seguida da função física (3,79), vitalidade (3,30), função social $(3,21)$, limitação no desempenho $(2,67)$ e saúde mental (2,63). No segundo momento, após a intervenção com a terapêutica não farmacológica de acupuntura e de eletroacupuntura, verificam-se estados muito próximos de saúde perfeita (médias com valores entre $1 \mathrm{e} 2$ ) com os seguintes resultados: função social (1,37); saúde mental (1,65); limitação no desempenho (1,74); dor física (1,79); função fisica $(1,91)$; e vitalidade $(1,98)$

TABELA 2 - Resultados das dimensões do SF-6D no primeiro e segundo momento de avaliação dos usuários de uma unidade de saúde familiar da região Norte de Portugal, 2017.

\begin{tabular}{lccc}
\hline Dimensões & $\begin{array}{r}\text { Primeiro } \\
\text { momento }\end{array}$ & $\begin{array}{r}\text { Segundo } \\
\text { momento }\end{array}$ & $\mathrm{P}^{+}$ \\
\hline Função fisica & $3,7 \pm 1,3$ & $1,9 \pm 1,1$ & 0,094 \\
$\begin{array}{l}\text { Limitação no } \\
\text { desempenho }\end{array}$ & $2,6 \pm 1,0$ & $1,7 \pm 0,8$ & 0,134 \\
Função social & $3,2 \pm 1,2$ & $1,3 \pm 0,9$ & 0,016 \\
Dor física & $4,7 \pm 1,1$ & $1,7 \pm 1,1$ & 0,595 \\
Saúde mental & $2,6 \pm 1,3$ & $1,6 \pm 0,8$ & 0,010 \\
Vitalidade & $3,3 \pm 1,2$ & $1,9 \pm 0,9$ & 0,061 \\
\hline
\end{tabular}

SF-6D, Short Form 6 Dimensions. Valores expressos em médiaıdesvio-padrão; tteste t de student.

Quando comparadas as várias dimensões do SF-6D nos dois momentos, constatamos diferenças em todas as dimensões com maior relevância para a dor física, cuja média passou de 4,72 para 1,79. A intervenção com a terapêutica não farmacológica de acupuntura e de eletroacupuntura melhorou a perceção da qualidade de vida relacionada com a saúde dos usuários que participaram no estudo.

Relativamente à presença de dor, os 43 (100\%) usuários referiram presença de dor, sendo que, no segundo momento, os resultados permitem-nos inferir que ocorreu uma redução acentuada no número de usuários (23; 53.5\%) que referiram a presença de dor. No que concerne à localização da dor, no primeiro momento, foi possivel verificar que 17 (39.5\%) usuários referiram que a área do corpo zona lombar mais perna, foi a área onde a presença de dor foi mais frequente, enquanto no segundo momento, essa área passou apenas a ser referida por dois (4.7\%) usuários.

A severidade da dor diminuiu do primeiro momento $34,9 \%$ dos doentes classificou a severidade máxima da dor em oito), para o segundo momento $(44,2 \%$ dos doentes classificou a severidade máxima da dor em zero. 
Numa análise mais pormenorizada na Tabela 3. verificamos que os usuários submetidos à intervenção com terapêutica não farmacológica de acupuntura e de eletroacupuntura, no segundo momento apresentam médias mais baixas nos domínios "severidade da dor" $(6,1)$ e "interferência dor no funcionamento diário" (7,9), relativamente ao primeiro momento, cujos valores eram de 22,4 e 43.9. Observaram-se diferenças do primeiro momento para o segundo momento de avaliação, quer no domínio severidade da dor, quer no domínio interferência da dor no funcionamento diário.

TABELA 3 - Resultados do BPI no primeiro e segundo momento de avaliação dos usuários de uma unidade de saúde familiar da região Norte de Portugal, 2017.

\begin{tabular}{|c|c|c|c|}
\hline Item & $\begin{array}{l}\text { Primeiro } \\
\text { momento }\end{array}$ & $\begin{array}{l}\text { Segundo } \\
\text { momento }\end{array}$ & $\mathbf{P}^{+}$ \\
\hline $\begin{array}{l}\text { BPI-3 - intensidade da } \\
\text { sua dor no seu máximo }\end{array}$ & $7,5 \pm 1,8$ & $2,9 \pm 3,0$ & 0,029 \\
\hline $\begin{array}{l}\mathrm{BPI}-4 \text { - intensidade da } \\
\text { sua dor no seu mínimo }\end{array}$ & $4,3 \pm 1,7$ & $1,0 \pm 1,4$ & 0,051 \\
\hline $\begin{array}{l}\text { BPI-5 - intensidade da } \\
\text { sua dor em média }\end{array}$ & $5,4 \pm 1,7$ & $1,8 \pm 1,9$ & 0,541 \\
\hline $\begin{array}{l}\text { BPI- } 6 \text { - intensidade da } \\
\text { sua dor neste preciso } \\
\text { momento }\end{array}$ & $5,1 \pm 2,3$ & $0,3 \pm 0,8$ & 0,341 \\
\hline $\begin{array}{l}\text { BPI-9a - interferência - } \\
\text { atividade geral }\end{array}$ & $6,7 \pm 2,3$ & $1,6 \pm 2,3$ & 0,002 \\
\hline $\begin{array}{l}\text { BPI-gb - interferência - } \\
\text { disposição }\end{array}$ & $6,1 \pm 2,8$ & $1,2 \pm 2,3$ & 0,031 \\
\hline $\begin{array}{l}\text { BPI-9c - interferência - } \\
\text { capacidade para andar a pé }\end{array}$ & $6,0 \pm 3,0$ & $1,0 \pm 2,2$ & 0,017 \\
\hline $\begin{array}{l}\text { BPI-9d - interferência - } \\
\text { trabalho normal }\end{array}$ & $7,0 \pm 2,2$ & $1,5 \pm 2,4$ & 0,053 \\
\hline $\begin{array}{l}\text { BPI-ge - interferência } \\
\text { - relações com outras } \\
\text { pessoas }\end{array}$ & $5,4 \pm 3,0$ & $0,9 \pm 2,0$ & 0,043 \\
\hline $\begin{array}{l}\text { BPI-gf - interferência - } \\
\text { sono }\end{array}$ & $7,7 \pm 2,2$ & $0,9 \pm 1,6$ & 0,023 \\
\hline $\begin{array}{l}\text { BPI-9g - interferência - } \\
\text { prazer de viver }\end{array}$ & $5,4 \pm 3,1$ & $0,5 \pm 1,9$ & 0,199 \\
\hline \multicolumn{4}{|l|}{ Subescalas } \\
\hline Severidade da dor (4 itens) & $22,4 \pm 6,7$ & $6,1 \pm 6,7$ & 0,069 \\
\hline Interferência da dor (7 itens) & $43,9 \pm 14,7$ & $7,9 \pm 13,4$ & 0,008 \\
\hline
\end{tabular}

BPI, Inventário Resumido da Dor. Valores expressos em média \pm desvio-padrão; theste t de student.
Quanto à interferência funcional da dor, avaliada por uma escala constituida por sete itens: atividade geral, humor, mobilidade, trabalho, relações pessoais, sono e prazer de viver, cuja classificação varia de zero a 10, as médias no segundo momento decresceram substancialmente, em todos os itens que integram a avaliação da interferência funcional, nomeadamente, nas relações pessoais, sono e com o prazer de viver. No primeiro momento de avaliação, 28 pacientes $(65,1 \%)$ referiam tomar medicamentos analgésicos e anti-inflamatórios, enquanto que no segundo momento, apenas sete (16,3\%) usuários referem tomar medicamentos analgésicos e anti-inflamatórios. No que respeita à avaliação dos custos diretos em saúde relacionados com medicação, para alívio e controlo da dor e tratamentos de fisioterapia realizados pelos usuários, foi constatado que, relativamente à medicação prescrita para alívio e controlo da dor, no primeiro momento, o valor de 449,43 euros, tendo passado no segundo momento, para 170,17 euros. No primeiro momento, os custos com a realização dos tratamentos de fisioterapia foram de 1.200,00 euros, tendo passado no segundo momento para 750 Euros. Relativamente à eficácia quanto ao alívio da dor na última semana, através da terapêutica não farmacológica de acupuntura e de eletroacupuntura, no segundo momento de avaliação, a maior parte dos usuários 23 (53.5\%) referiram alivio total da dor, seguidos por nove $(20,9 \%)$ usuários que referiram que o alivio da dor se situou em 90\%.

\section{Discussão}

No estudo de Stival et al. (8), cujo objetivo era avaliar a eficácia da acupuntura no tratamento da fibromialgia, onde participaram 36 usuários em regime ambulatório, 21 foram submetidos a uma sessão de acupuntura tradicional chinesa e 15 doentes a um procedimento de acupuntura placebo (acupuntura sham). Foi utilizada a escala visual analógica para avaliação da dor, antes e após a sessão de acupuntura, e os resultados mostraram que a diferença na amplitude de variação da escala visual analógica entre os grupos, foi melhor no grupo experimental e que a acupuntura foi eficaz na redução imediata da 
dor nos usuários com fibromialgia. Outro estudo, realizado para avaliar os efeitos da acupuntura no tratamento da dor lombar em gestantes (9), no segundo e no terceiro trimestre de gravidez, em 56 usuárias grávidas com dor lombar, quase-experimental, antes e depois, mostrou que, após o tratamento com acupuntura, houve redução estatisticamente significativa $(p<0,001)$ da dor lombar, tendo concluído que a acupuntura apresentou efeitos na redução e no alivio da dor. Também o estudo, sobre a ação da aurículoacupuntura em pessoas com dor crônica na coluna vertebral: ensaio clínico randomizado (6), realizado numa amostra de 110 usuários (randomizados em três grupos) verificou que houve redução significativa da incapacidade nos grupos tratado e placebo entre as avaliações inicial e final $(p<0,05)$ e entre as avaliações inicial e follow up $(p<0,05)$. $\mathrm{Na}$ avaliação final, o grupo tratado apresentou melhorias na incapacidade, comparando o grupo placebo ao grupo de controlo $(p<0,05)$, aumento na temperatura média tecidular da região dorsal entre as avaliações inicial e follow up no grupo tratado e de controlo $(p<0,05)$, tendo concluido que a auriculoacupuntura foi eficaz para reduzir a incapacidade e aumentar a temperatura tecidular em pessoas com dor crónica na coluna vertebral.

No estudo que Grillo et al. (16) efeturam, foram realizadas avaliações dos aspetos psicológicos de 40 usuárias com disfunção temporomandibular, sendo avaliadas com o questionário do Research Diagnostic Criteria for Temporomandibular Disorders, no pré e pós-tratamento, e uma escala visual analógica, que avaliava a expectativa (negativa ou positiva) do tratamento. No pré-tratamento mostrou melhorias na intensidade da dor crónica (53,3\%), na depressão estado grave (11,1\%), na somatização estado grave (16,7\%) e, ainda, uma correlação negativa $(p<0,05)$ entre a expectativa de tratamento e de somatização, nas usuárias em tratamento com acupuntura, tendo concluido que alguns dos aspetos psicológicos (depressão e somatização) melhoraram após curto periodo de tratamento.

De forma análoga, o estudo, sobre avaliação do controle da dor miofascial crônica em cabeça e pescoço utilizando a técnica nova crânio acu- puntura de Yamamoto et al. (17), realizado junto de 20 usuários com cefaleia e dor no pescoço há mais de três meses, mostrou que a média atribuida à dor no inicio do tratamento foi 7.9 e após oito semanas reduziu-se para 0,8 e concluiu que os resultados apontam para a eficácia da acupuntura para o alivio de sintomas álgicos principalmente quando se pretende a diminuição imediata da dor.

No estudo de Hadianfard et al. (18), que avaliou o efeito da acupuntura na dor e na qualidade de vida em usuários com estenose da coluna vertebral lombar, realizado em 24 usuários que apresentavam sintomas de claudicação neurogénica, selecionados aleatoriamente e submetidos a 10 sessões de acupuntura, sendo, a dor e a qualidade de vida, avaliadas antes e imediatamente após a intervenção e seis semanas depois, utilizando uma escala visual analógica e o Short Form (SF-6D)-36, os resultados mostraram uma diminuição significativa ( $p<0,001$ ) nos scores médios da escala visual analógica antes e imediatamente após a intervenção, assim como o score médio semanas depois $(p=0,01)$ e nas seis dimensões do SF-6D foram associados melhorias significativas imediatamente após a acupuntura $(p<0,05)$. Esses autores concluiram que a acupuntura teve um efeito significativo de curto prazo na dor e na qualidade de vida, sendo associada à melhoria da dor e à melhoria da qualidade de vida e o efeito foi persistente seis semanas após a intervenção. $O$ mesmo refere Lemmon (19), no seu estudo sobre acupuntura e dor, onde é apoiado o uso da acupuntura para o tratamento da dor lombar crónica.

O estudo de Christian et al. (20) refere que em ambientes de ambulatório, a acupuntura melhora a dor lombar crónica levemente à moderadamente e melhora mais a função, mais do que o tratamento convencional ou nenhum tratamento e melhora a qualidade de vida mais do que nenhum tratamento. É referido, ainda, que usuários em tratamento de reabilitação hospitalar e acupuntura para lombalgia crónica apresentam melhorias ligeiramente maiores do que individuos envolvidos somente na reabilitação tradicional.

A acupuntura é, assim, um dos instrumentos mais proficuos na medicina complementar (21), 
e por isso, a sua utilização em algumas patologias dolorosas traz um benefício relevante, notoriamente favorável ao individuo uma vez que é eficiente na dor, mostrando-se como uma conduta em crescimento contínuo.

Os resultados obtidos nesta pesquisa evidenciam diferenças estatisticamente significativas entre o primeiro momento e o segundo momento de avaliação, que se traduziu no aumento da qualidade de vida relacionada com a saúde, com reflexo na melhoria significativa do controlo da dor dos usuários com sindrome vertebral com irradiação, resultante da intervenção com a terapêutica não farmacológica de acupuntura e de eletroacupuntura, influenciando positivamente a severidade da dor, a interferência no funcionamento diário e no consumo de tratamentos e medicamentos para o alívio da dor.

Podemos dizer que a utilização da terapêutica não farmacológica de acupuntura e de eletroacupuntura parece diminuir a severidade da dor e a interferência da dor no funcionamento diário, contribuindo para uma melhoria da qualidade de vida dos usuários com dor crónica.

Torna-se claro, também, que a terapêutica não farmacológica de acupuntura e de eletroacupuntura contribuiu para a redução do consumo de anti-inflamatórios e analgésicos e, consequentemente, para uma diminuição nos custos diretos relacionados com a aquisição desses medicamentos e, não menos relevante, a ocorrência de efeitos adversos comum dessas prescrições. A utilização da terapêutica não farmacológica de acupuntura e de eletroacupuntura no controlo da dor crónica parece traduzir um ganho em custos diretos na saúde com redução significativa nos custos com a medicação e os tratamentos de fisioterapia.

Os resultados desta pesquisa, apontam para a necessidade de se continuar a investigar a relação existente entre a utilização da terapêutica não farmacológica de acupuntura e de eletroacupuntura na gestão da dor crónica.

A grande limitação desta pesquisa tem a ver com a população que esteve na base do estudo, isto é, ser limitada somente a uma unidade de saúde familiar da região Norte de Portugal.
Consideramos que, no futuro, seria importante o desenvolvimento de estudos longitudinais em amostras mais alargadas, com grupo de controlo, com inclusão de usuários com outras patologias, que sedimentem na comunidade científica a eficácia desta terapêutica.

\section{Notas}

Parte deste trabalho é resultado da dissertação de mestrado de um dos autores (EFC), intitulada "Terapêutica não farmacológica de acupuntura e eletroacupuntura no controlo da dor crónica em doentes com sindrome vertebral com irradiação", apresentada na Universidade de Trás-os-Montes e Alto Douro, Portugal.

\section{Apoio financeiro}

Este estudo não recebeu apoio financeiro de fontes externas.

\section{Declaração de conflito de interesses}

Os autores declaram não haver conflitos de interesses relevantes ao conteúdo deste estudo.

\section{Contribuições dos autores}

Todos os autores fizeram contribuições substanciais para concepção, ou delineamento, ou aquisição, ou análise ou interpretação de dados; e redação do trabalho ou revisão critica; e aprovação final da versão para publicação.

\section{Disponibilidade dos dados e responsabilidade pelos resultados}

Todos os autores declaram ter tido total acesso aos dados obtidos e assumem completa responsabilidade pela integridade destes resultados.

\section{Referências}

1. IASP. Pain Terms, A Current List with Definitions and Notes on Usage (Part III). Classification of Chronic Pain. 2nd ed. Seattle: ISAP Press; 1994.

2. Pereira DTS, Andrade LL, Agra G, Costa MML. Condutas terapêuticas utilizadas no manejo da dor em oncologia. Cuidado é Fundamental. 2015:7(1):1883-90. http:// dx. doi.org/10.9789/2175-5361.2015.v7i1.1883-1890 
3. Direção Geral de Saúde. Programa Nacional para a Prevenção e Controlo da Dor. Ministério da Saúde. Lisboa; 2017 [cited 2019 Ago 20]. Available from: https://www. dgs.pt/documentos-e-publicacoes/programa-nacional-para-a-prevencao-e-controlo-da-dor-pdf.aspx

4. Almeida DC, Kraychete DC. Dor lombar - uma abordagem diagnóstica. Rev dor. 2017:18(2):173-7. https:// doi.org/10.5935/1806-0013.20170034.

5. Stump GA, Kobayashi R, Campos WA. Lombociatalgia. Rev dor. 2016;17(Suppl1):63-6. http://dx.doi. org/10.5935/1806-0013.20160051

6. Moura CC, lunes DH, Ruginsk SG, Souza VHS, Assis BB, Chaves ECL. Action of ear acupuncture in people with chronic pain in the spinal column: a randomized clinical trial. Rev Latino-Am Enfermagem. 2018;26:e3050. https://doi.org/10.1590/1518-8345.2678.3050

7. Park J, Clement R, Hooyman N, Cavalie K, Ouslander $\mathrm{J}$. Factor structure of the Arthritis-Related Health Belief instrument in ethnically diverse community-dwelling older adults with chronic pain. J Community Health. 2015:40(1):7381. https://doi.org/10.1007/s10900-014-9898-7

8. Stival RSM, Cavalheiro PR, Stasiaka CES, Galdinoa DT, Hoekstra BE, Schafranskib MD. Acupuntura na fibromialgia: um estudo randomizado-controlado abordando a resposta imediata da dor. Rev Bras Reumatol. 2014:54(6):4316. http://dx.doi.org/10.1016/j.rbr.2014.06.001

9. Martins ES, Tavares TMC, Lessal PRA, Aquino PS, Castro RCM, Pinheiro AKB. Tratamento com acupuntura: avaliação multidimensional da dor lombar em gestantes. Rev Esc Enferm USP. 2018;52:e03323. http://dx. doi. org/10.1590/S1980-220X2017040303323

10. Muñoz-Ortego J, Solans DM, Carrion C. Medical indications for acupuncture: Systematic review. Med Clin. 2016;147(6):250-6. https://doi.org/10.1016/j.medcli.2016.02.029

11. World Health Organization. Who traditional medicine strategy 2014-2023. Suiça: World Health Organization. 2013; [cited 2020 Jun 12]. Available from: https://apps.who.int/iris/bitstream/handle/10665/92455/978924150609o_eng.pdfijsessioni$\mathrm{d}=\mathrm{A} 542064 \mathrm{BE} 31 \mathrm{CBO}$ E903FA3967FoF8F7C? sequence $=1$

12. Maciocia G. A prática da medicina chinesa: tratamento de doenças com acupuntura e ervas chinesas. São Paulo: Roca; 1996

13. Silvério-Lopes S. Eletroacupuntura e Eletropuntura. In: Silvério-Lopes, editor. Analgesia por Acupuntura [Internet]. Curitiba: Omnipax Editora Ltda; 2013 [cited 2019 Jan 12]. p. 63-80. Available from: http://omnipax. com.br/livros/2013/ANAC/anac-Livro.pdf

14. Azevedo F, Pereira A, Dias C, Agualusa L, Lemos L, Romão J et al. Tradução, adaptação cultural e estudo multicêntrico de validação de instrumentos para rastreio e avaliação do impacto da dor crónica. Dor [Internet]. 2007 [cited 2020 Abr 7]:15(4):6-37. Available from: https://www.aped-dor.org/socios/material_bibliografico/diversos_Questionarios_Dor-Rev_DOR_Volume15-n4-2007.pdf
15. Ferreira P. Ferreira L. A medição de preferências em saúde na população portuguesa. Revista Portuguesa de Saúde Pública [Internet]. 2006 [cited 2020 Abr 7]; 24(2):5-14. Available from: https://www.uc.pt/feuc/ pedrof/docs/Publicacoes/AN200605.pdf

16. Grillo CM, Canales GLT, Wada RS, Barbosa CMR, Fausto BF, Sousa MLR. Aspetos psicológicos de pacientes com disfunção temporomandibular: avaliações pós tratamento com acupuntura. Rev dor. 2015;16(2):114-8. https://doi.org/10.5935/1806-0013.20150022.

17. Correia LMF, Albertil D, Lopes SS. Avaliação do controle da dor miofascial crônica em cabeça e pescoço utilizando a técnica Nova Crânio Acupuntura de Yamamoto em acompanhamento durante 8 semanas. Rev dor. 2015;16(2):81-5. https://doi.org/10.5935/1806$\underline{0013.20150016}$

18. Hadianfard MJ, Aminlari A, Daneshian A, Safarpour AR. Effect of Acupuncture on Pain and Quality of Life in Patients with Lumbar Spinal Stenosis: A Case Series Study. J Acupunct Meridian Stud. 2016;9(4):178-82. https://doi.org/10.1016/i.jams.2015.11.032

19. Lemmon R. Acupuncture for pain: 7 questions answered. J Fam Pract. 2018; 67(4):224-30.

20. Christian V, Mark Z, Jeremy O, Brett D. Is acupuncture beneficial for the treatment of lower back pain? Evidence-Based Practice. 2018;21(5):E3-E5. https://doi. org/10.1097/01.EBP.0000545128.56114.1e

21. Zuo Z, Huang P, Jiang Y, Zhang Y, Zhu M. Acupuncture attenuates renal interstitial fibrosis via the TGF- 8 / Smad pathway. Mol Med Rep. 2019;20(3):2267-75. https://doi.org/10.3892/mmr.2019.10470

\section{Elisabete Fernandes Carvalho}

Mestre em Enfermagem Comunitária pela Universidade de Trás-os-Montes e Alto Douro, em Vila Real, Portugal; Enfermeira Especialista no Agrupamento de Centros de Saúde do Cávado III - Barcelos/Esposende, Portugal.

\section{Dinis Rodrigues Brito}

Mestre em Medicina pela Faculdade de Medicina da Universidade do Porto, no Porto, Portugal; Médico de Medicina Geral e Familiar no Agrupamento de Centros de Saúde Cávado I, em Braga, Portugal.

Isabel Maria Antunes Rodrigues da Costa

\section{Barroso}

Mestre em Ciências de Enfermagem pela Universidade do Porto, no Porto, Portugal; Professora Adjunta na Universidade de Trás-os-Montes e Alto Douro, em Vila Real, Portugal. 


\section{Maria João Filomena dos Santos Pinto}

\section{Monteiro}

Doutora em Ciências Humanas e Sociais - Ciências da Educação pela Universidade de Trás-os-Montes e Alto Douro, em Vila Real, Portugal. Professora Coordenadora na Universidade de Trás-os-Montes e Alto Douro, em Vila Real, Portugal.

\section{Vitor Manuel Costa Pereira Rodrigues}

Doutor em Ciências Biomédicas pela Universidade do Porto, no Porto, Portugal; Professor Coordenador Principal na Universidade de Trás-os-Montes e Alto Douro, em Vila Real, Portugal.

\section{Endereço para correspodência}

Vitor Manuel Costa Pereira Rodrigues

Universidade de Trás-os-Montes e Alto Douro

Escola Superior de Saúde

Quinta de Prados, Complexo Desportivo

$5000-801$

Vila Real, Portugal

Os textos deste artigo foram revisados pela Poá Comunicação e submetidos para validação do(s) autor(es) antes da publicação. 